\title{
Excitation of the porous silicon photoluminescence by a multiphoton vibronic process
}

\author{
J. Diener, M. Ben-Chorin, D. I. Kovalev ' , S. D. Ganichev' ${ }^{1}$, F. Koch \\ Technische Universität München, Physik-Department E16, D-85747 Garching, Germany
}

\begin{abstract}
We study the emission of photons from porous $\mathrm{Si}$ after high-level vibrational excitation in the $\mathrm{Si}-\mathrm{O}$ absorption band using a pulsed $\mathrm{CO}_{2}$ laser. Narrow resonances in the efficiency of light generation are discovered at $1030 \mathrm{~cm}^{-1}$ and $1084 \mathrm{~cm}^{-1}$. For excitation at $1030 \mathrm{~cm}{ }^{-1}$ the familiar photoluminescence band of porous Si appears. Time-resolved pump-to-probe experiments show the same decay times for the photo and the IR-induced luminescence. We suggest a microscopic process responsible for the light emission.
\end{abstract}

Keywords: Luminescence; Optical properties; Silicon

In recent work we have observed light emission from porous silicon (PS) after pulsed IR excitation [1]. An intense pulsed $\mathrm{CO}_{2}$ laser has been used to excite resonantly the IR active stretching mode of the $\mathrm{Si}-\mathrm{O}$ bonds. An excitation within the broad $\mathrm{Si}-\mathrm{O}$ absorption band results always in a generation of visible light. Nevertheless two resonances in the efficiency of light generation are observed at $1084 \mathrm{~cm}^{-1}$ and $1030 \mathrm{~cm}^{-1}$ (Fig. 1). This two modes differ by their spectral and temporal response. The first one has a slower time response and its spectrum show no features typical for the photoluminescence (PL) of PS. The light emission in this mode of excitation results from multiphoton excitation of the Si-O bonds, which do not couple efficiently with the electronic states of PS. Under the other mode of excitation $\left(1030 \mathrm{~cm}^{-1}\right)$, the light emission characteristics are similar to the usual PL of PS. In this paper we want to emphasize this mode and to discuss its properties in detail.

Samples used in the experiment were conventional microporous Si prepared by electrochemical etching from p-type (2-5 $\Omega \mathrm{cm}),(100)$-oriented $\mathrm{Si}$ wafers. The electrolyte was ethanoic HF in a 1:1 volume mixture of pure $\mathrm{C}_{2} \mathrm{H}_{5} \mathrm{OH}$ and concentrated aqueous HF. Typical sample thicknesses were 2-5 $\mu \mathrm{m}$. To provide a moderate oxidation they were heated at $200^{\circ} \mathrm{C}$ under ambient conditions for different times.

For the experiments the sample was mounted in an evacuated chamber ( $\sim 1$ mbar). A high power, pulsed, TEAmode $\mathrm{CO}_{2}$ laser was used as IR excitation source. The pulse

\footnotetext{
' Permanent address: A.F. Ioffe Physicotechnical Institute, Russian Academy of the Sciences, St. Petersburg, 194021, Russia.
}

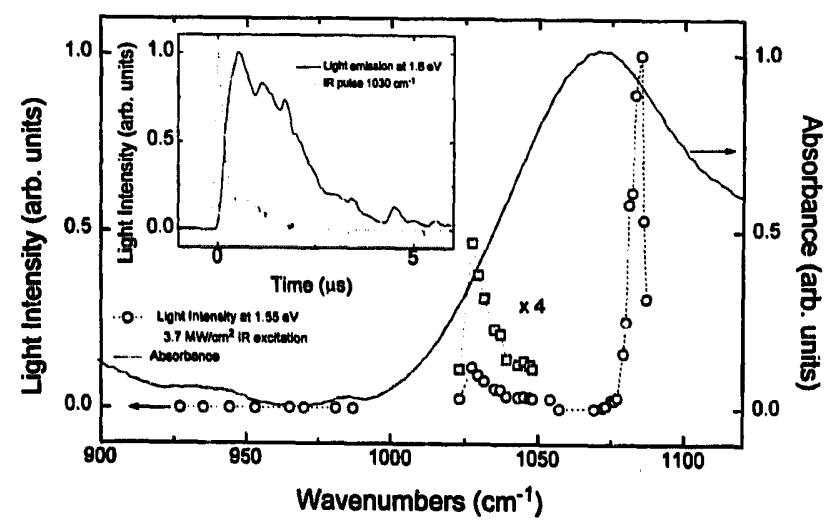

Fig. 1. Light emission, detected at $1.55 \mathrm{eV}(\mathrm{O})$, as a function of the IR photon energy for fixed IR intensity of $I_{\mathrm{IR}}=3.7 \mathrm{MW} \mathrm{cm}^{-2}$. The maximum at $1030 \mathrm{~cm}^{-1}$ is enlarged by a factor of four $(\square)$. The solid line represents the IR absorbance of the porous Si layer. Inset: the temporal behavior of the optical signal detected at $\left.1.8 \mathrm{eV} \mathrm{(-}{ }^{-}\right)$. The dotted line shows the shape of the IR excitation pulse $\left(1030 \mathrm{~cm}^{-1}, 2 \mathrm{MW} \mathrm{cm}^{-2}\right)$.

enters the chamber through a thick Ge window that filters visible and near infrared light. The PL was detected by a fast photomultiplier and Ge detector. Spectral resolution is achieved with a monochromator. All PL spectra were corrected for the spectral response of the optical system. The experiments were performed at room temperature.

The dominant sample response to the IR pulse is a weak luminescence signal extending in time for a few $\mu$ s (inset Fig. 1). For the $1030 \mathrm{~cm}^{-1}$ excitation, the luminescence peaks around $300 \mathrm{~ns}$ after the IR pulse, and decays with a time constant of $1.4 \mu \mathrm{s}$ thereafter. The spectrum of the generated light has a peak around $1.6 \mathrm{eV}$ (Fig. 2, circles), super- 


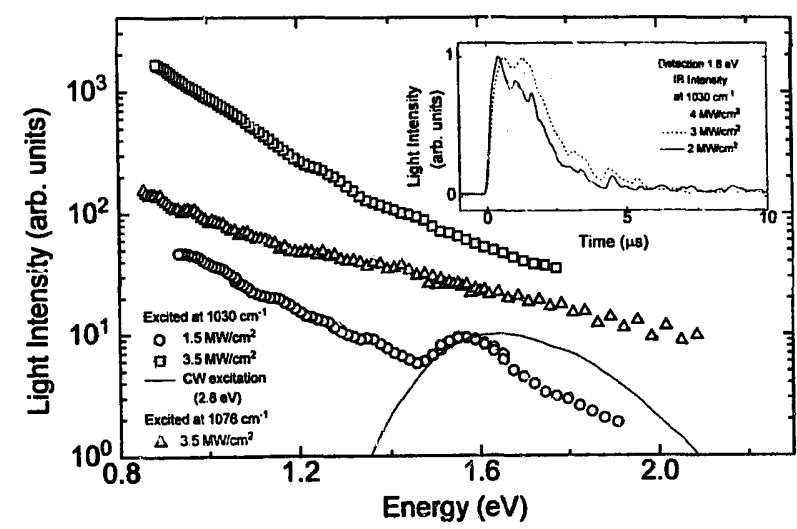

Fig. 2. IR-induced luminescence spectra at different excitation intensities for two different IR photon energies. The spectra are shifted along the $y$ axis for clarity. Inset: time response of the optical signal at $1.8 \mathrm{eV}$ for different IR pulse iniensities (at $1030 \mathrm{~cm}^{-1}$ ). All curves in the inset are normalized to $I$ at their maximum.

imposed on a broad, monotonically decreasing background. After correcting for the background response, the spectral distribution of the peak follows more or less the usual PL of the sample, when excited with the $2.8 \mathrm{eV}$ line of a HeCd laser (Fig. 2, solid line). Note that the energy position of the peak $(1.6 \mathrm{eV})$ corresponds to more than 12 photons of the incident IR radiation. With increasing IR intensity the peak is washed out (Fig. 2, squares), and the spectral behavior resembles that of the light emitted under the $1084 \mathrm{~cm}^{-1}$ excitation (Fig. 2, triangles). In the same time the dynamics of the IRinduced light changes. The inset in Fig. 2 shows the temporal behavior of the emitted light, detected within the red peak at $1.8 \mathrm{eV}$, for different excitation intensities. At low excitation levels, for which a peak in the emission spectrum is clearly resolved, the emission rises fast and reaches its maximum less than $0.5 \mu$ s after the IR pulse (inset Fig. 2, solid line). An increase of the incident IR intensity leads to a slower luminescence signal (inset Fig. 2, dashed line). It seems that the signal has fast and slow components, with comparable intensities, giving rise to the multiple maxima structure observed in the time response. Finally, at higher IR intensities, the dynamics are very similar to that observed for the $1084 \mathrm{~cm}^{-1}$ excitation (inset Fig. 2, dotted line). The emission reaches a maximum after a delay of $\sim 2 \mu \mathrm{s}$. The fast component is weak, attributing only a slight step on the increasing edge of the signal. A further increase of the intensity does not lead to a change in the temporal or spectral response of the emitted light. This is in agreement with the behavior of the light emitted under the $1084 \mathrm{~cm}^{-1}$ excitation, where both the spectrum and the time response of the emitted light are independent of the exciting IR intensity [1].

The peak observed in the spectral behavior of the emitted light under $1030 \mathrm{~cm}^{-1}$ excitation reminds the usual PL of PS. This suggests that we might have succeeded to excite the usual PS luminescence with an IR pulse. However, the temporal response of the IR-induced signal is very different from the dynamics of the PL decay. A decay time of $1.4 \mu \mathrm{s}$ at $1.8 \mathrm{eV}$ is much faster than that found for the photoexcited luminescence signal [2]. If we couple to the electronic states responsible for the PL of PS one has to explain this discrepancy between the time scales. In order to do that we investigate the behavior of the photoexcited PL lifetime following the IR excitation. The arrangement is identical to pump-toprobe experiments, where the time delay between the emission of two pulsed lasers is tuned. The $\mathrm{CO}_{2}$ radiation is used as the pump. $\mathrm{A} \mathrm{N}_{2}$ laser $(3.68 \mathrm{eV}, 300 \mathrm{ps}$ ) is used to excite the luminescence of PS, which is monitored as a probe. This allows us to study the influence of IR excitation on the usual photoexcited PL.

Fig. 3 (left part) shows the change in the decay of the PL when the excitation with the $\mathrm{N}_{2}$ laser occurs at different delay times after the IR pulse. After the IR laser pulse the intensity of the PL maximum drops and the decay time becomes faster with respect to the unperturbed case. With increasing delay time the PL maximum rises and the decay time becomes slower. The change of the lifetime as a function of the delay between the two lasers is shown in the inset of Fig. 3 (right part). The lifetime becomes longer and longer and after about $1 \mathrm{~ms}$ it returns to its initial value (without $\mathrm{CO}_{2}$ laser excitation). This is easily explained in terms of heating of the sample by the $\mathrm{CO}_{2}$ laser pulse. For higher temperatures (above room temperature) shorter lifetimes are expected [2]. We have calibrated the temperature increase responsible for the shortening of the lifetimes in a d.c.-type experiment on the same sample. The change of the sample temperature as a function of time after the $\mathrm{CO}_{2}$ laser pulse (as deduced from both the lifetimes and the maximum of the PL) is shown in the right part of Fig. 3. Approximately $5 \mu \mathrm{s}$ after the IR pulse the sample has a temperature of $\sim 200^{\circ} \mathrm{C}$, which is in agreement with other experiments performed to determine the increase of the sample temperature [1]. Then, the temperature decays with a time constant of the order of $100 \mu \mathrm{s}$.

The $\mathrm{CO}_{2}$ laser pulse is therefore a tool to heat the sample within a short time (of the order of $\mu \mathrm{s}$ ), and the sample then stays hot for a relatively long time. This allows one to investigate the influence of a fast heat pulse on the PL. This is
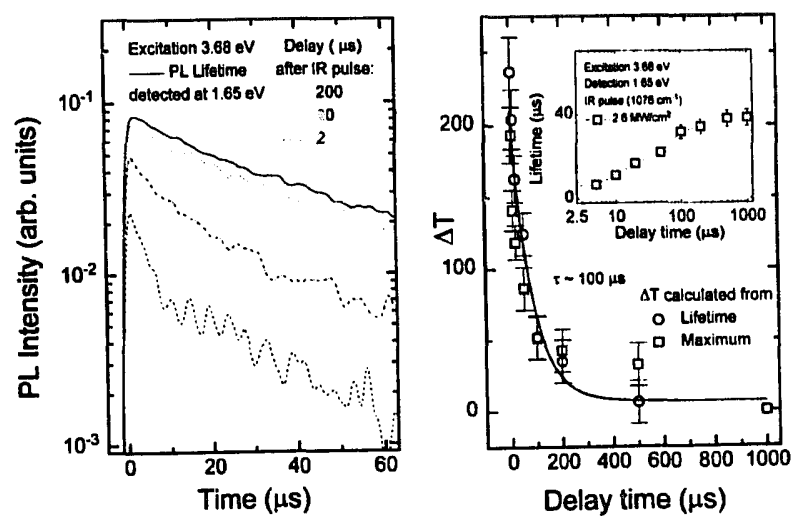

Fig. 3. Left: the time dependence of the luminescence intensity, detected at $1.65 \mathrm{eV}$. The different curves correspond to an excitation by the $\mathrm{N}_{2}$ laser at different delays after the IR pulse. Right: the change of the sample temperature after the IR pulse as a function of the delay time. Inset: lifetimes deduced from the PL decay times vs. the delay time after the IR pulse. 

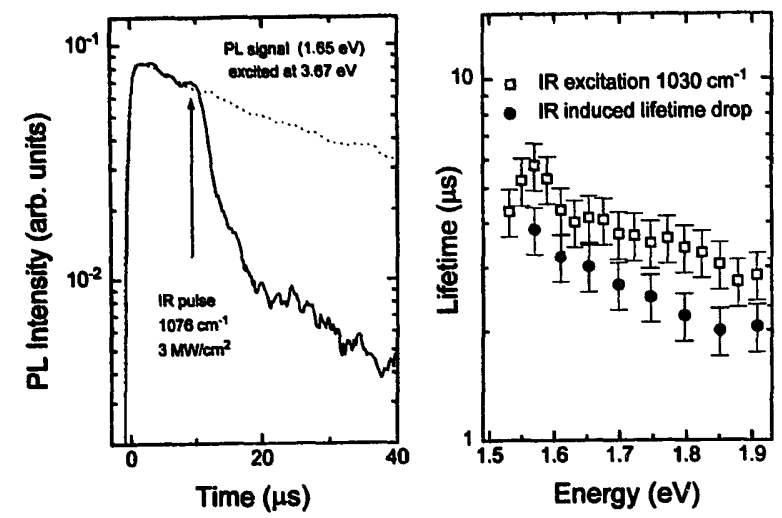

Fig. 4. Left: the temporal response of the PL when the IR pulse $\left(1076 \mathrm{~cm}^{-1}\right.$, $3 \mathrm{MW} \mathrm{cm}^{-2}$ ) is tuned after the $\mathrm{N}_{2}$ laser pulse, within the PL decay. Right: comparison between the characteristic time of the drop in the PL signal following the IR pulse $(\Theta)$ and the lifetimes of the IR-induced luminescence (ㅁ).

achieved by exciting with the IR pulse after the UV pulse, within the decay time of the PL. Before the IR pulse heats the sample the decay is identical to that of the unperturbed case (Fig. 4, left part). Following it, a fast drop of the PL is observed, with a decay time of $\sim 2.5 \mu \mathrm{s}$. After this drop a slow decay is observed, with a lifetime which is only slightly faster than seen for the unperturbed case.

The above result helps us to understand the question from which we started: what is the origin of the difference between the dynamics of the usual PL and the IR-induced (1030 $\mathrm{cm}^{-1}$ ) light emission. Our assumption is that by excitation with $1030 \mathrm{~cm}^{-1}$ we couple into the electronic states responsible for the usual PL. The problem is the difference in lifetimes. However, we have just shown that even the usual PL decays very fast while the IR pulse is on. Therefore, the same will happen for the IR-induced light. A comparison between the dynamics of the IR-induced $\left(1030 \mathrm{~cm}^{-1}\right)$ luminescence (taken $3 \mu$ s after the IR pulse) and that of the usual PL (at the same delay time with respect to the IR pulse) is given in the right part of Fiy. 4. Both the UV-excited luminescence and the IR-excited luminescence decay within the same time scale over the whole spectral region.

We now turn to discuss the observed results. The absorption of the IR photons by the Si-O boni results in an oscillation of these vibration modes. For an excitation at 1084 $\mathrm{cm}^{-1}$ more than 2 optical Si phonons are needed in order that a vibration would relax. Since the $1030 \mathrm{~cm}^{-1}$ excitation is near to resonance with 2 optical phonons in the nanocrystallites [3], this mode provides a better coupling between the surface oscillations and the Si core of the nanoparticles. As the dissipation time of phonons in the Si core is quite short, the whole crystallite is heated very fast. It is possible to describe this excitation as a fast heating step. Carriers, trapped on the surface, are then able to overcome energy barriers and to enter the luminescing particle. Once created, they recombine in the usual luminescence step.
We turn now to explore the short decay time of the signal. Usually the effective lifetime $\tau_{\mathrm{efr}}$ is described by:

$\frac{1}{\tau_{\text {eff }}}=\frac{1}{\tau_{\mathrm{r}}}+\frac{1}{\tau_{\mathrm{nr}}}$

where $\tau_{\mathrm{r}}$ and $\tau_{\mathrm{nr}}$ are the radiative and non-radiative lifetimes respectively. Both $\tau_{\mathrm{r}}$ and $\tau_{\mathrm{nr}}$ are temperature dependent. Furthermore, within the same model, the quantum efficiency $\eta_{\text {eff }}$ is:

$\eta_{\mathrm{eff}}=\frac{\tau_{\mathrm{nr}}}{\tau_{\mathrm{r}}+\tau_{\mathrm{nr}}}$

However, Eqs. (1) and (2) cannot explain the fast drop of the PL after the IR excitation, as well as the decrease of $\eta_{\mathrm{eff}}$ after the pulse by more than one order of magnitude while the lifetime of the PL after the IR pulse is only slightly changed. We explain the observed behavior by a simple approach, which regards PS as a granular material consisting of luminescing and dark crystallites. The first type are crystallites which do not contain a non-radiative center, and the dark ones have at least one killing state. We further assume $\tau_{\mathrm{nr}} \ll \tau_{\mathrm{r}}$, and that the transfer of excitation between crystallites is impossible. Therefore the probability for electron-hole pairs to recombine radiatively in such a dark crystallite is nearly zero. Luminescing crystallites are free of non-radiative centers, providing an opportunity for electron-hcle pairs to recombine radiatively. Consequently the PL intensity (quantum efficiency) is determined by the ratio between the density of these two types of crystallites and the observed lifetime is $\tau_{r}$. The IR excitation results in an increase of the number of dark crystallites either by creation of additional dangling bonds or by opening an additional nonradiative channel. This leads to a drop in the intensity of the emitted light, but only to a slight change in the lifetime afterwards. To resolve such a fast drop the non-radiative recombination time for the dark crystallites has to be at least as fast as the observed $\sim 2.5 \mu \mathrm{s}$ time of the drop.

The change in the ratio between luminescing and dark particles, caused by the IR radiation, also explains the properties of the IR $\left(1030 \mathrm{~cm}^{-1}\right)$ induced luminescence. In the same moment, when carriers are randomly injected into the crystallites by the heat pulse, the probability that this crystallite would become dark, due to non-radiative recombination, is increased. This is supported by the similarity between the decay times of the IR-induced, red-peaked light emission, and the drop time of the usual PL signal following the IR excitation. This is another evidence that both light emissions are similar in nature, manifested by the emission spectrum and the corresponding PL lifetimes.

Nevertheless, at high IR excitation intensities the nonradiative rate becomes so fast that the probability to excite a luminescing crystallite is small. Therefore, this mode of light generation is not efficient. This is in agreement with the change in the spectral response observed for high IR excitation levels, which does not show the red peak. 
Finally, we point out that the short non-radiative lifetime $<2.5 \mu$ s during the IR pulse also explains why a red peak is not observed under the $1084 \mathrm{~cm}^{-1}$ excitation. Since the heat transfer in this case is slow, of the order of a few $\mu$ s, the thermal injection rate of trapped carriers is so small compared with the non-radiative recombination that practically no red light can be observed.

To summarize we have shown that it is possible to excite luminescence in PS using IR pulse. The mechanism of this luminescence is similar to thermo-stimulated luminescence in glasses, in which fast heating results in light emission. The results of time-resolved studies show that PS behaves like a granular system of luminescing and dark crystallites.

\section{Acknowledgements}

We acknowledge support for this work by the BMFT. DK is an Alexander von-Humboldt Foundation fellow. SG thanks the DFG. JD is sponsored by Siemens AG in its SFE program.

\section{References}

[1] J. Diener, M. Ben-Chorin, D.I. Kovalev, S.D. Ganichev and F. Koch, Phys. Rev., B52 (1995) R8617.

[2] J.C. Vial, A. Bsiesy, F. Gaspard, R. Herino, M. Ligeon, F. Muller, R. Romestain and R.M. Macfarlane, Phys. Rev., B45 (1992) 14171.

[3] J.B. Renucci, R.N. Tyte and M. Cardona, Phys. Rev., Bll (1975) 3885; Z. Sui, P. Leong, I.P. Herman, G.S. Higashi and H. Temkin, Appl. Phys. Lett., 60 (1992) 2086. 